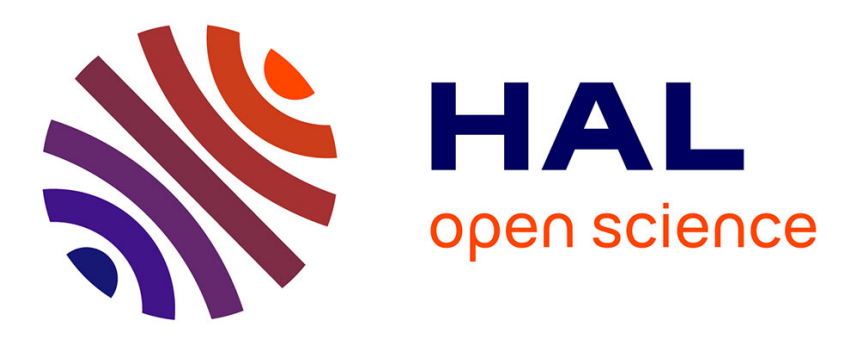

\title{
Les prud'hommes des campagnes. Fonctions et représentations des élites paysannes en Gascogne occidentale au XIIIe siècle
}

Frédéric Boutoulle

\section{- To cite this version:}

Frédéric Boutoulle. Les prud'hommes des campagnes. Fonctions et représentations des élites paysannes en Gascogne occidentale au XIIIe siècle . Le concept d'élites en Europe de l'Antiquité à nos jours, Mar 2011, Pessac, France. pp.47-65., 10.4000/books.msha.18841 . hal-01316286

\author{
HAL Id: hal-01316286 \\ https://hal.science/hal-01316286
}

Submitted on 20 May 2016

HAL is a multi-disciplinary open access archive for the deposit and dissemination of scientific research documents, whether they are published or not. The documents may come from teaching and research institutions in France or abroad, or from public or private research centers.
L'archive ouverte pluridisciplinaire HAL, est destinée au dépôt et à la diffusion de documents scientifiques de niveau recherche, publiés ou non, émanant des établissements d'enseignement et de recherche français ou étrangers, des laboratoires publics ou privés. 


\title{
Les prud'hommes des campagnes. Fonctions et représentations des élites paysannes en Gascogne occidentale au XIIIe siècle
}

\author{
Frédéric Boutoulle \\ Université Bordeaux Montaigne / UMR Ausonius 5607 \\ Labex Sciences Archéologiques de Bordeaux
}

Contrairement à la bourgeoisie des villes, cette force sociale investissant communes et municipalités urbaines naissant aux XII ${ }^{\mathrm{e}}$ siècle puis s'affirmant au XIII ${ }^{\mathrm{e}}$ siècle, les élites paysannes sont longtemps restées méconnues des historiens, ce qui a contribué à entretenir la réputation d'espaces ruraux apathiques, socialement peu discriminés et dont la population est massivement soumise aux nouvelles formes de servage des XII et XIIIe siècles (vilainage anglais, remensa catalane, questalité gasconne) ${ }^{1}$. Ce n'est qu'assez récemment que des travaux ont mis en lumière des élites roturières au sein de la société rurale du Moyen Âge central avec, par exemple, les vavasseurs normands étudiés par Matthieu Arnoux ou les pages, dits aussi "hommes des casaux» de la Gascogne méridionale, mis en lumière par Benoît Cursente ${ }^{2}$. La sensibilité des historiens aux travaux des sociologues œuvrant sur les élites contemporaines est pour une large part responsable de ces nouvelles orientations permettant de mieux comprendre la complexité des sociétés médiévales au-delà des habituelles divisions ternaires. Le choix du thème élitaire aux XXVII journées de Flaran en 2005 ( $L$ Les élites rurales dans l'Europe médiévale et moderne ») témoigne de la réception, chez les médiévistes ruralistes, de questionnements jusqu'alors surtout confinés aux groupes nobiliaires ou aux sociétés urbaines ${ }^{3}$. On ne peut pas non plus être insensible au fait que cet aggiornamento se soit déroulé en Gascogne orientale, une région où, sur ce thème, les renouvellements vont bon train grâce aux travaux de B. Cursente, Mireille Mousnier,

\footnotetext{
${ }^{1}$ FELLER Laurent, Paysans et seigneurs au Moyen Âge, VIII -XVe siècles, Paris, Colin, 2007, p. 165-191.

${ }^{2}$ ARnouX Matthieu, «Classe agricole, pouvoir seigneurial et autorité ducale. L'évolution de la Normandie d'après le témoignage des chroniqueurs Xe-XIIe siècles », Le Moyen Âge, 98, 1992, p. 35-60 ; ID., "Rustici et homines liberi. Où sont passés les serfs normands ? ", Mélanges de l'école française de Rome. Moyen Âge, $\mathrm{t}$. 112, 2, 2000, p. 563-577 ; ID., «Les paysans et le duc : autour de la révolte de 996 », in La Normandie vers l'an Mil, Société d'Histoire de la Normandie, Rouen, 2000, p. 105-111; ID., «Remarques sur les fonctions économiques de la communauté paroissiale (Normandie, XIIe-XIII' siècles)», in BARTHELEMY, D. et MARTIN, J.-M. dir. Liber largitorius. Études d'histoire médiévale offertes à Pierre Toubert par ses élèves, Genève, 2003, p. 413-430 ; ID., "Pour une économie historique de la dîme ", in VIADER, R. dir. La dîme dans l'Europe médiévale et moderne, Actes des XXXe Journées internationales d'histoire de l'abbaye de Flaran, 3-4 octobre 2008, Toulouse, 2010, p. 145-161. CuRSENTE Benoît, « La société rurale gasconne au miroir des cartulaires (XIe-XIII ${ }^{\mathrm{e}}$ s.), Notables du fisc ou paysans ? », in Villages et villageois au Moyen Age, XXI congrès de la SHMES, Paris, 1992, p. 53-65 ; ID., "Puissance, liberté, servitude. Les casalers gascons au Moyen Âge ", Histoires et sociétés rurales, 6, 2e semestre 1996, p. 31-50 ; ID., Des maisons et des hommes, La Gascogne médiévale (XIe-XVe siècle), Toulouse, 1998 ; ID., « De la queste à la questalité : l'avènement d'un servage institutionnalisé en Gascogne (XII ${ }^{\text {-XIII }}$ siècles) ", in BouRIN, M. et FREEDMAN, P. dir, La servitude dans les pays de la Méditerranée occidentale chrétienne au XIIe siècle et au-delà : déclinante ou renouvelée ? Actes de la table ronde de Rome, 8 et 9 octobre 1999, Rome, Mélanges de l’Ecole française de Rome, 112, 2, 2000, p. 941-960 ; ID., «Les Abbadies ou Abbayes laïques : dîme et société dans les pays de l'Adour $\left(\mathrm{XI}^{\mathrm{e}}-\mathrm{XVI}^{\mathrm{e}}\right.$ siècles), Annales du Midi, CXVI, 247, juillet-septembre 2004, p. 285-305 ; ID., " Le cartulaire du chapitre de Dax et la société des laïcs ", in CABANOT, J. et MARQUETTE, J.-B. L'Église et la société dans le diocèse de Dax aux XIe-XIIe siècles. Journée d'étude sur le Livre rouge de la cathédrale de Dax, Dax 1er mai 2003, Dax, Amis des anciennes églises des Landes \& Comité d'études sur l'histoire et l'art de la Gascogne, 2004, p.73-87.

${ }^{3}$ MENANT François et Jean-Pierre JESSEnNE dir., Les Élites rurales dans l'Europe médiévale et moderne, Actes des XXVII Journées Internationales d'Histoire de l'abbaye de Flaran, 9, 10, 11 septembre 2005, Toulouse, Presses universitaires du Mirail, 2007.
} 
Roland Viader, Anne Berdoy ou d'Hélène Couderc-Barraud ${ }^{4}$. Cette contrée présente en effet des sources normatives précoces, fors et chartes de coutumes, reconnaissant aux « voisins » un droit à la violence légitime, une juridiction au-delà de l'espace domestique ou même un partage de juridiction, leur conférant collectivement une puissance politique et sociale en face de laquelle le pouvoir seigneurial, vicomtal ou comtal doit composer 5 .

Les prud'hommes des campagnes de la Gascogne occidentale du XIII siècle (Bordelais, Bazadais, Dacquois, fig. 1), auxquels seront consacrées les lignes qui suivent, offrent bien des points communs avec les groupes dont il vient d'être question ${ }^{6}$. S'il méritent qu'on s'intéresse plus particulièrement à eux, c'est que l'on bénéficie à leur propos d'une exceptionnelle conjonction de sources pour comprendre sur quoi, sur quelles fonctions, se fonde la domination sociale des élites traditionnelles non nobles en pays d'habitat dispersé, et comment, à l'aide de quel type d'argumentaire, ils sont amenés à la défendre.

Fig.1

\section{Une exceptionnelle conjonction de sources}

Quoique non exclusivement, l'appellation de prud'hommes pour désigner des groupements de notables paysans du XIIIe siècle est surtout présente dans les rôles de la chancellerie anglaise, car le roi d'Angleterre est aussi, depuis 1154, duc d'Aquitaine et de

\footnotetext{
${ }^{4}$ MousNiER Mireille, «Jeux de mains, jeux de vilains. Hommages et fidélités serviles dans le Languedoc médiéval (XII ${ }^{e}$ XIII ${ }^{\mathrm{e}}$ siècles ", Histoire et Sociétés rurales, n¹4, 2000, p.11-54 ; EAD., L'abbaye cistercienne de Grandselve et sa place dans la société et l'économie méridionales (XIIe-XIVe siècles), Toulouse, Presses universitaires du Mirail, 2006 ; VIADER Roland, « Le servage en Gascogne et en Languedoc aux XIII" et XIVe siècles. Quelques éléments de réflexion ", in CARRIER, N. dir., Nouveaux servages et société en Europe (XIII XXe siècle). Actes du colloque de Besançon (4-6 octobre 2007), Caen, Association d'histoire des sociétés rurales, 2010, p. 41-65 ; BERDOY Anne, «Abbayes laïques et domenjadures : l'habitat aristocratique en haut Béarn », dans Résidences aristocratiques, résidences du pouvoir entre Loire et Pyrénées, $X^{e}-X V^{e}$ siècles, Actes du colloque de Pau, 3-5 octobre 2002, Archéologie du Midi médiéval, suppl. n 4, 2006, p. 65-103 ; EAD. « Des abbayes laïques, fossiles d'un peuplement per casalem intégrés dans des castra », Annales du Midi, juilletseptembre 2008, tome 120, n²63, p.337-358; COUDERC-BARRAUD Hélène, La violence, l'ordre et la paix. Résoudre les conflits en Gascogne du XIe au début du XIIIe siècle, Toulouse, Presses universitaires du Mirail, 2008 ; EAD., «Humbles et violence légale. Quelques cas gascons, XII ${ }^{\mathrm{e}}$-début XIII ${ }^{\mathrm{e}}$ siècle », in FolLAIN, A. dir., La violence et le judiciaire. Discours, perceptions pratiques, Rennes, 2008, p. 31-46 ; EAD., « Résistances antiseigneuriales en Gascogne : pactes et affrontements (XIIe-début du XIII e siècle), in BRUNET S. et BRUNEL Gh. dir. Haro sur le seigneur. Les luttes antiseigneuriales dans l'Europe médiévale et moderne. Actes des XXIXe journées internationales d'Histoire de Flaran 5-6 octobre 2007, Toulouse, Presses Universitaires du Mirail, 2009, p.111-123.

${ }^{5}$ EAD., La violence l'ordre et la paix. Résoudre les conflit en Gascogne du XI au début du XIII siècle, Toulouse, 2008, p. 226-240 ; EAD; «Humbles et violence légale. Quelques cas gascons, XII ${ }^{\mathrm{e}}$-début XIII ${ }^{\mathrm{e}}$ siècle », art. cit., p. 31-46.

${ }^{6}$ Boutoulle Frédéric, "L'enquête de 1236-1237 en Bordelais », in PeCout, Th. Quand gouverner c'est enquêter. Les pratiques politiques de l'enquête princière. Occident, XIII ${ }^{e}-X V^{e}$ siècles (actes du colloque UMR Telemme, Aix-Marseille 19-21 mars 2009), De Boccard, Paris, 2010, p. 117-131; ID., «"Il est un meilleur roi que le roi d'Angleterre". Note sur la diffusion et la fonction d'une rumeur dans la paysannerie du Bordelais au XIII ${ }^{\mathrm{e}}$ siècle », in BILlore, M. et SORIA, M., La rumeur au Moyen Âge. Du mépris à la manipulation, $V^{e}-X V^{e}$ siècle, Rennes, PUR, 2011, p. 279-291; ID., «Pouvoirs et protagonistes territoriaux dans le domaine ducal gascon : l'Entre-deux-Mers bordelais d'après l'enquête de 1236-1237», in CASTELNUOVO, G. et ZoRZI, A., Les pouvoirs territoriaux en Italie centrale et dans le sud de la France. Hiérarchies, institutions, langages $\left(X I I^{e}-X I V^{e}\right.$ siècles), colloque organisé par École Française de Rome en collaboration avec l'Università du Firenze et l’Université de Savoie, Rome I ${ }^{\mathrm{er}}$ décembre 2006 (à paraître).
} 
Gascogne : Patent rolls, Close rolls et, à partir de 1272, Gascon Rolls ou Rôles gascons7. Dans ces rôles sont consignés des centaines de mandements, ordres de paiement, quittances adressés aux officiers du souverain, ainsi qu'aux communautés et seigneurs gascons. Dans ces séries, l'expression probi homines a deux usages spécifiques : il s'agit tantôt d'individus appelés à rendre sous serment un témoignage ou une expertise dans le cadre d'une procédure judiciaire (à l'instar des legales homines de visneto anglais); tantôt de représentants de communautés d'habitants avec qui le roi est en relation directe, qu'il s'agisse de collèges composés d'un nombre de prud'hommes défini comme il s'en trouve dans les villes dotées d'une organisation municipale ou bien de représentants informels, ce qui est le cas dans les campagnes du domaine ducal.

À propos de ce deuxième groupe, les mandements issus de la chancellerie anglaise permettent de cerner les fonctions des prud'hommes, ce que le roi en attend, et donc d'éclairer les fonctions de médiateur du dominium royal. Cette documentation éclaire aussi les formes de représentations pratiquées au sein des communautés rurales en l'absence d'institutions collectives comparables aux municipalités urbaines. Cependant, et c'est le principal problème posé par ce type de sources, les prud'hommes auxquels le roi s'adresse ne sont pas nominativement désignés, ce qui ne permet pas d'établir des listes et de mener des enquêtes prosopographiques en croisant les informations avec d'autres sources.

Les chartes de coutumes et de franchises constituent un autre bon révélateur de l'objet de cette recherche. Le XIII siècle est justement la période de mise par écrit des franchises locales, en faveur de communautés d'habitants vivant en ville, dans les castra, mais aussi, et c'est moins connu, pour quelques zones rurales, à la manière des " confédérations » villageoises picardes ${ }^{8}$. Nous avons ainsi conservé les coutumes de l'Entre-deux-Mers bordelais, consignées dans le procès verbal de l'enquête de 12361237 dont nous reparlerons et qui concernent une quarantaine de paroisses rurales de l'Entre-deux-Mers bordelais faisant partie du domaine ducal ${ }^{9}$. D'autres chartes de coutumes ont été accordées à de semblables groupements de paroisses rurales par le prince Édouard, le fils d'Henri III, dans le sud du Dacquois, en 1255 (Maremne, Marensin, Gosse, Seignanx, Labenne, Laluque ${ }^{10}$ ). Cependant, s'ils fixent les droits et devoirs des habitants, éclairent parfois le rôle de ceux de ces notables qui ont des fonctions de médiation sous des appellations diverses (anciens, procureurs, jurats), ou

\footnotetext{
${ }^{7}$ Michel Francisque éd., Rôles Gascons (1242-1254), t.1, Paris, Imprimerie nationale, Documents inédits de l'histoire de France, 1885 (désormais RG) ; BEMONT Charles, éd., Rôles Gascons (1254-1255), supplément au tome I, Paris, Imprimerie nationale, Documents inédits de l'histoire de France, 1896 (désormais RG).

${ }^{8}$ Fossier Robert, Chartes de coutume en Picardie (XIe-XIII ${ }^{e}$ siècle), Paris, Bibliothèque nationale, Documents inédits pour l'histoire de France, 1974, p. 114, BouRIN Monique, DuRAND Robert, Vivre au village au Moyen Age : les solidarités paysannes du XIe au XIIIe siècle, Paris, Rennes, 1984, rééd. 2000, p. 188-190.

9 Petit cartulaire de La Sauve-Majeure, Bibliothèque municipale de Bordeaux, ms 770, p. 126-135 (copie achevée avant le 12 octobre 1270). Édition partielle dans Gallia Christiana, t. II, instr. col. 289) et SMANIOTTO, Michel, Le cartulaire de La Sauve-Majeure, mémoire dactylographié, s.d., déposé à la Bibliothèque municipale de Bordeaux, ${ }^{\circ} \mathrm{B} 48$ (édition. sans notes ni tableau de la tradition). Édition de la version gasconne de l'enquête dans «Coutumes et privilèges de l'Entre-deux-Mers », éd. DELPIT, Jules Archives Historiques du département de la Gironde, t. III, Bordeaux, 1861-1862, p. 101-127 (édition de D), nXXXII à XXXVIII, avec réorganisation des documents de la première partie, classés chronologiquement par l'éditeur avec adjonctions de lettres d'Henri III du 3 août $1236\left(n^{\circ}\right.$ XXXIV et XXXV), du 28 novembre 1236 ( ${ }^{\circ}$ XXXVII) et du vidimus du procès verbal de l'enquête ( ${ }^{\circ} \mathrm{XXXVIII)}$. Nous renvoyons à nouvelle édition de la version latine que nous venons d'achever et qui sera publiée prochainement (désormais Enquête et coutumes Entre-deux-Mers).

${ }^{10}$ BEMONT, Charles. op. cit., n 4395 .
} 
mettent en lumière quelques procédures de désignation des représentants, ces textes présentent de sérieux problèmes à l'historien: en Dacquois, les seules coutumes conservées de la liste ci-dessus (Maremne, Marensin) sont connues par des versions écrites tardives, un constat qui vaut aussi pour les coutumes des castra et des bourgs, dont les versions écrites qui nous sont parvenues témoignent d'adjonctions des XIVe, XVe et $\mathrm{XVI}^{\mathrm{e}}$ siècles ${ }^{11}$. Le repérage des informations les plus anciennes n'y est pas simple alors qu'il s'agit d'une étape incontournable pour mieux faire résonner les informations extraites des autres types de sources du XIIIe siècle.

Ces élites rurales apparaissent dans les enquêtes dont il est fait un plus large recours au XIII ${ }^{\mathrm{e}}$ siècle. La plus importante est celle de 1236-1237. Diligentée par le roi Henri III, à la suite de plainte de ses sujets sur les exactions commises par ses baillis en Entre-deuxMers à l'encontre des populations locales et des médiateurs traditionnels du dominium royal, cette enquête mobilise une centaine de jurés désignés par leurs noms, venus d'une vingtaine de paroisses de l'Entre-deux-Mers ducal déposer sous serment sur les exactions dont leurs paroisses sont les victimes, ainsi que sur les droits et devoirs auxquels ils sont normalement tenus. Autre enquête précieuse pour notre sujet, celle qui est connue sous le nom de Reconnaissances féodales de 1274 (Recognitiones feodorum in Aquitania), par lesquelles plus de 500 seigneurs et représentants de communautés de la Gascogne anglaise, du Bordelais au Labourd, déclarent sous serment ce qu'ils tiennent du roi et à quels devoirs ${ }^{12}$ : ces aveux dévoilent également une partie du patrimoine foncier des élites rurales, tout au moins ce qu'ils déclarent tenir du roi, ainsi que les devoirs collectifs des communautés dont ils sont les médiateurs.

Enfin, la documentation foncière de ce siècle, faite d'actes de la pratique, apporte de précieux compléments. Cette documentation est d'abord composée de cartulaires, comme le Second cartulaire de Sainte-Croix de Bordeaux (169 actes datés de 1233 à 128313), celui de l'infirmier de La Sauve Majeure (37 actes entre 1222 et $1255^{14}$ ), le Liber Aureus de la cathédrale de Bayonne pour les actes du XIIIe siècle qu'il contient (5 actes concernant le Dacquois méridional entre 1233 et $1266^{15}$ ) ainsi que des pièces isolés dans les liasses des fonds de La Sauve et Sainte-Croix de Bordeaux aux archives départementales de la Gironde. Cet ensemble de sources permet de suivre à la trace quelques uns des prud'hommes qui se sont manifestés à l'occasion des enquêtes, d'appréhender leur patrimoine, d'en savoir plus sur leurs autres fonctions et sur leurs sociabilités. Les éléments de patrimoine appartenant à des individus reconnus comme des prud'hommes ou des jurés de l'enquête de 1237 révèlent des ensemble variés,

\footnotetext{
${ }^{11}$ Les coutumes du Marensin sont insérés dans le Coutumier de Dax (4 articles commençant par « Note que es costume en Marensin », ABBADIE François, éd., Le livre noir et les établissements de Dax, Bordeaux, 1902, art. 196-199, p. 57-58). Ce long coutumier a été compilé par un notaire de l'officialité, Jean de la Porta, après 1468 , à partir notamment d'un coutumier plus ancien, et perdu depuis, que le compilateur appelle «le libre de la biele » ou le « libre de la ciutat». Pour celles de Maremne, OLCE (d') éd., « Matériaux pour servir à l'histoire des Landes. Statut de la vicomté de Maremne », Bulletin de la société de Borda, 1882-1883. Ces coutumes sont datées du « 15 juin 1300 », mais ce texte est écrit dans un français du XVI ${ }^{\mathrm{e}}$ siècle. Voir aussi HIRIGoyen, Francis, Histoire de la baronnie de Labenne-Cap-Breton et de la vicomté de Maremne, Pau, 2001

${ }_{12}$ BEMONT Charles, éd., Recueil d'actes relatifs à l'administration des rois d'Angleterre en Guyenne au XIIIe siècle (Recogniciones feodorum in Aquitania), Paris, Imprimerie nationale, Documents inédits de l'histoire de France, 1914 (désormais RF).

${ }^{13}$ DRouyn Léo, éd. «Second cartulaire de l'abbaye Sainte-Croix de Bordeaux », Archives Historiques de la Gironde, t. 27, 1892 (désormais Sainte-Croix II) p.159-292.

${ }^{14}$ AD Gironde, H4.

${ }^{15}$ BIDACHE Paul, éd., Le livre d'or de Bayonne, Pau, 1906.
} 
composés de tenures et d'alleux leur permettant d'avoir des tenanciers et l'accès aux vacants ou padouens, sur lequel ils peuvent exercer une forme de contrôle.

$\mathrm{Au}$ total, il ressort de cette documentation que le terme de prud'homme n'est pas exclusif pour désigner les élites paysannes du domaine ducal avec qui le roi-duc est en relation. Ils sont aussi considérés comme des « hommes francs » ou des « hommes libres » en vertu des franchises dont ils se revendiquent, "bons hommes », "voisins » en Dacquois, voire "anciens ». Il faut aussi dire que probi homines n'est pas non plus réservé aux élites roturières. L'expression qui reflète autant un idéal de vie qu'un statut reconnu est aussi adoptée par l'aristocratie, jusqu'au roi de France Louis IX lui-même d'après une confidence de son biographie Joinville ${ }^{16}$. C'est précisément parce que cet idéal de prud'homie transcende les habituelles catégories de la société et qu'il est partagé aussi bien par des roturiers que par des chevaliers qu'il est à même de rendre compte de la perméabilité des lignes de démarcations au sein des sociétés rurales.

\section{La médiation du dominium ducal}

Nous disposons malheureusement de peu de données sur les arbitrages rendus par les notables paysans et sur leur rôle dans la résolution des conflits intra-communautaires, qui relèvent pourtant des attributions de cette catégorie d'élites traditionnelles, depuis les boni homines Languedociens jusqu'aux goði Islandais ${ }^{17}$. La documentation est moins laconique sur leurs fonctions de médiation avec les pouvoirs englobants.

Habituellement, on considère que la médiation de l'autorité seigneuriale est exercée par des agents, des ministériaux nommés ou titulaires d'un office, voire par des vassaux issus de l'aristocratie et ayant reçu délégation sur une fraction du territoire seigneurial. La médiation qu'exercent les prud'hommes ne rentre dans aucune de ces catégories. Leur fonction de répartiteurs des exigences ducales met plutôt en lumière une autre forme de médiation, appuyée sur les élites paysannes locales et, semble-t-il, non formalisée par une délégation en bonne et due forme. Pour autant qu'on puisse le voir, les demandes qu'ils relaient concernent trois domaines distincts.

Ils sont d'abord chargés de la répartition des hébergements réclamés par le duc et ses agents, comme le montre ce passage du procès verbal de l'enquête de 1237 concernant les coutumes du pays.

« $\mathrm{Si}$, dans sa mission de poursuite des ennemis de la paix, à la nuit tombée, [le roi] ne peut se rendre dans une ville ou dans un château pour y trouver des choses à acheter, il aura l'hébergement sur les paysans des villages environnants, quels que soient leurs seigneurs, et selon une répartition opérée par les bons hommes responsables des hébergements entre les nombreux villages et entre chaque paysan, afin que personne ne soit lésé, à l'exclusion des lieux jouissant d'une immunité ecclésiastique ou d'un privilège du seigneur roi $»^{18}$.

\footnotetext{
${ }^{16}$ Jean de Joinville, Histoire de saint Louis, PAuPHILET Albert éd. Historiens et chroniqueurs du Moyen Âge, Paris, Gallimard, Bibl. le Pléiade, vol. III, 1952, p. 205.

17 Bourin Monique, Villages médiévaux en Bas-Languedoc : genèse d'une sociabilité. Xe-XIVee siècle, Paris, 1987 ; Вүоск, Jesse, L'Islande des Vikings, Paris, Aubier, 2001.

${ }^{18}$ Enquête et coutumes Entre-deux-Mers : Haberet albergagiam in agricolis, villarum forentium cuiuscumque essent agricole et per bonos homines terre albergatores dimidientur, per multas villas et per singulos agricolas, ita quod nulius guaretur, exceptis tamen locis que immunitate ecclesiastica gaudere solent, vel privilegio domni regis.
} 
L'aubergade dont il est question, ou droit de gîte, consiste à répondre aux besoins en nourriture et en fourrage du duc, de ses agents et de leur troupe ${ }^{19}$. Ce vieux droit régalien largement diffusé au sein de la société seigneuriale sous des appellations et des modalités diverses, donne lieu à des extorsions dont les contribuables cherchent à se prémunir. Le même texte détermine en effet que " pour que les hébergements du seigneur roi $(\ldots)$ on ne doit pas tuer d'autre animal qu'un porc, un bélier, une oie, ou une poule», un principe de modération qui, à en juger par les exemples recueillis dans les dépositions, est loin d'être respecté par les prévôts du roi et son sénéchal.

Leur rôle est similaire dans la collecte des questes royales. Ces contributions directes sont assimilées à tort à ce que donnent à leurs seigneurs les questaux, c'est-à-dire les hommes qui ont versé dans une des formes de la nouvelle servitude des XIIe et XIIIe ${ }^{20}$. Dans ce dernier cas, la queste des questaux (dite aussi taille) est individuelle, aléatoire et peu à peu associée à des incapacités juridiques (mainmorte, attache à la glèbe). Au contraire, les questes royales acquittées entre la fête de Saint-Michel et Toussaint sont payées par les hommes francs du domaine royal, parallèlement avec d'autres prestations comme le service militaire ou l'obligation d'assister aux cours de justice devant le représentant du roi, autant de formes de service dont le caractère public n'échappe à personne. Il s'agit ni plus ni moins d'une forme d'impôt direct, levé dans la Gascogne ducale plus précocement que dans le domaine royal des capétiens où ce n'est qu'avec la croisade de saint Louis de 1248 et l'aide requise en cette occasion que les recettes extraordinaires commencent à l'emporter sur les ressources ordinaires ${ }^{21}$.

Ainsi, le 31 août 1224, Henri III demande-t-il aux prud'hommes du pays de Gosse, en Dacquois (probis hominibus de Govossa), de percevoir les "rentes " qu'ils doivent de leurs terres pendant les quatre prochaines années à compter de la prochaine fête de saint Michel, afin d'en verser le produit au maire et aux prud'hommes de Bayonne, pour les aider à fortifier la ville ${ }^{22}$. Le 26 septembre 1242, Henri III mande aux prud'hommes de 19 communautés du Dacquois occidental de livrer les quêtes devant lui être rendues au terme de la prochaine fête de Saint-Michel, dans les mains de l'évêque de Dax, à qui le roi avait donné l'ordre de les recevoir pour en verser le produit à de ses créanciers, Gaillard Colomb ${ }^{23}$. En Entre-deux-Mers bordelais, si le procès verbal de l'enquête de 1236-1237 n'évoque nulle médiation des prud'hommes dans ce domaine, on sait par deux actes fonciers consignés dans le second cartulaire de Sainte-Croix de Bordeaux, datés de 1275 et 1279, pour des biens situés à Lignan, que l'acquéreur doit « payer tous

\footnotetext{
${ }^{19}$ BRÜHL Carl Richard, Fodrum, gistum, servitium régis. Grundlagen des Königtums im Frankreich und in den Nachfolgestaaten Deutschland, Frankreich und Italien vom 6. bis zur Mitte des 14. Jahrhunderts. Böhlau, Köln und Graz 1968.

${ }^{20}$ FELLER, Laurent, op.cit. , CuRSENTE Benoît, Une histoire de la questalité. Serfs et libres dans le Béarn médiéval, Pau, 2011.

${ }^{21}$ SiVERY Gérard, Les Capétiens et l'argent au siècle de saint Louis, Essai sur l'administration et les finances royales au XIIIe siècle, Lille, 1995.

${ }^{22}$ Patent Rolls 1216-1225, 1224, p. 467.

${ }^{23} R G \mathrm{n}^{\circ}$ 485. Rex probis hominibus suis de Goosa, de Seint-Gorbs, de Saz, de Engomer, de Sabussa, de Lorgon, de Sont, de Sancto Vincentio, de Tossa, de Saubion, de Angossa, de Benessa, de la Farina, de Soston, de Marensin, de Masanges, de la Luca, de Pontons, et de Seins Gyrons salutem. Mandamus vobis quod omnes redditus quos nobis debitis ad hunc terminum Sancti Michaelis anno etc. XXVI, reddatis venerabili patri Aquensi episcopo, cui injuximus quod redditus illos a vobis recipiat, et eos faciat habere Gailardo Columbo in partem solutionis debitorum que ei debemus.
} 
les ans six deniers de quête générale aux prud'hommes de Lignan, à la fête de SaintMichel $»^{24}$.

Les modalités de levée de cette queste royale à la collecte de laquelle les prud'hommes sont associés, nous échappent en grande partie. Si l'on en croit l'absence de montant déterminé dans les mandements adressés aux prud'hommes du Dacquois en 1224 et 1242, comme la précision d'une levée de 4 ans dans l'un de ceux-là, ces questes collectives conservent elles aussi un caractère aléatoire. Cependant, les choses ne tardent pas à être fixées et à s'organiser. En 1237, les jurés qui déposent devant les envoyés du roi, et parmi lesquels se comptent certainement quelques uns des répartiteurs, prétendent qu'en Entre-deux-Mers, le montant de la queste est égal à 40 livres. Cette demande d'abonnement de la queste est entérinée puisqu'en 1274, le même montant de 40 livres est signalé dans la reconnaissance collective des hommes francs de l'Entre-deux-Mers ${ }^{25}$, alors que ceux du Bazadais doivent collectivement 20 livres $^{26}$. Ces jalons textuels dessinent une autre évolution. Dans un premier temps puisque une somme globale est attendue de chaque groupements de paroisses (40 livres en Entredeux-Mers, 20 livres en Bazadais), la queste doit être considérée comme un impôt par répartition, à propos duquel on suppose que les prud'hommes de chaque paroisse répartissent le fardeau entre les différents foyers fiscaux et en tenant compte du niveau de richesse de chacun. Le principe est en effet exposé pour la levée d'une taille dans une localité dépendante de l'abbaye Sainte-Croix, à Macau, en 1255, selon «le pouvoir et la richesse » des hommes tenants feu-vif» ou «selon que les maisons sont grandes ou petites »27. Dans les coutumes de Labouheyre, en Dacquois, au nom du même principe de solidarité fiscale, «le fort supporte le faible ${ }^{28}$. Dans un second temps, le système par quotité semble s'imposer. C'est que le l'on déduit de la reconnaissance collective du 23 mars 1274 qui précise la quotité de vingt-et-une paroisses et six lieux-dits de l'Entre-deuxMers ducal, avec une modulation de deux sous à quatre livres, de celles des hommes francs du Bazadais, ou encore des deux actes de 1275 et 1279 indiquant le montant de la quote part à la queste du roi évoquée ci-dessus ${ }^{29}$.

\footnotetext{
${ }^{24}$ Sainte-Croix II, p.159-292, $\mathrm{n}^{\circ} 163, \mathrm{n}^{\circ} 217$, p. 208.

${ }^{25} R F, \mathrm{n}^{\circ} 537$.

${ }^{26} R F, \mathrm{n}^{\mathrm{os}} 244,245,246,247,248,249,250,251,338$. Et $R F, \mathrm{n}^{\circ} 338$, qui signale qu'il s'agit d'une queste.

${ }^{27}$ Sainte-Croix II, ${ }^{\circ} 291$, p. 266-269. Et disso meis que li avandeit home et lur her dongan fromentada a l'abat et aus monges avandeitz, cada an, entre la Sent-Miqueu et la Totz-Sentz; so es a ssaber, per cada foc biu que en la bila de Macau sere o aure estat lo plus d'aquet an, I boisset de froment, so es a saber d'artant bet et artant bon froment cum comunau trobera hom a bendre au port a Bordeu, et a la mesura que lo dia que aquesta carta fo feyta era corsabla et bendabla a Bordeu; loquau boisset deven metre mesurat et gardar dins la gleysa de Macau per que contrast ni dompta no pusca estre; et aquet blat lo celerers Senta Crois o sos comandamentz. deu talhar et partir, ab dos prohomes de Macau que y apere, sobre us homes que fucs bius tendran, aissi cum es d'avandeit, segont lo poder et la riquesa et la praubressa deus medis homes quauque horas bulha, dins l'avantdeit terme, et, la talha feyta, deven li homes aver pagat lo blat tot lo darer dins l'avantdeit terme de la Totz-Sent. Et per medissa maneyra, deu los dar cada maysons, en que fuc no tendra home, mechs boisset de forment la majer, et las autras, per arradon que seran grans o paucas, a conoguda deu celarer o de son comandament ab los prohomes de Macau qu'i apera. Pero tant exceptât, que li hom, qui fuc biu $i$ tengos, ave una mayson en que tengos son blat o son bin, o sa ordilha o sos baissetz o sas autras causas, sénés que fuc biu no y tengos, aquera deu estre quitta deu mechs Boisset.

${ }^{28}$ MARQUETTE Jean-Bernard, "Les coutumes de Labouheyre (Herbafauere) », dans Un pays dans sa langue. Le gascon dans l'ensemble d'oc. Actes du colloque de Sabres, sous la présidence de Pierre Bec, 9, 10 octobre 2004, Travaux et colloques scientifiques, n5, Parc naturel régional des Landes de Gascogne, 2006, p. 107140.

${ }^{29}$ Sainte-Croix II, n 163 (deven pagar VI $d$. de questa generau an per an, a la sent Miqueu, aux prohomes de Linhan) et $\mathrm{n}^{\circ} 217$, p. 208 (12 deniers par an au titre de la « queste du roi ».
} 
La répartition des questes et des hébergements entre les différents foyers d'une communauté paroissiale est certainement une entreprise difficile, plus particulièrement en contexte de tension démographique, ce qui semble être le cas dans ces campagnes durant la première moitié du XIIIe siècle. Si le thème du dépeuplement de la région revient si fréquemment dans les dépositions réunies dans l'enquête de 1237, comme dans les demandes de confirmation des franchises des prud'hommes de l'Entre-deux-Mers adressées au roi Jean en $1214^{30}$, c'est que le départ des habitants vers les villes des environs dotées de franchises comme Bordeaux, ou vers de plus lointains horizons ibériques, complique singulièrement le travail des répartiteurs s'ils ne sont pas compensés par le croît démographique ou par l'apport migratoire. Que survienne une famine, comme celle qui est attestée dans plusieurs sources régionales en 1235 , et c'est de fragiles équilibres sociaux qui se trouvent menacés ${ }^{31}$. L'affaire est d'autant plus délicate qu'un degré de responsabilité semble lier les répartiteurs à ces levées. C'est la raison pour laquelle les coutumes tardives de Maremne autorisent les voisins qui collectent et apportent la queste, à prendre des gages des gages sur les autres voisins de leur paroisse ne pouvant ou ne voulant pas payer la queste.

Dans le domaine militaire enfin. Une série de mandements à l'ost adressés par le roi, le 21 juillet 1230 aux prud'hommes des francs du Bazadais et de l'Entre-deux-Mers ${ }^{32}$, le 6 juillet 1242 aux prud'hommes du Marensin et de Gosse ${ }^{33}$, et le 9 décembre 1253 aux prud'hommes d'une dizaines de communautés rurales du Dacquois (dont Laharie, Marensin, Maremne Gosse, Seignanx, Labenne, Laluque), montrent qu'ils peuvent être chargés de relayer les ordres de convocation des milices rurales et de les conduire en un lieu déterminé pour se joindre à l'ost royal ${ }^{34}$. Cependant, quand ils éclairent les modalités du service militaire des paysans, telles qu'on les trouve dans les coutumes de l'Entre-deux-Mers de 1237 comme dans les prescriptions concernant les habitants des

\footnotetext{
${ }^{30}$ HARDY Thomas Duffus, éd., Rotuli litterarum patentium in turri Londinensi asservati, Londres 1835, vol. I, pars I, 1201-1226, p. 112b (La Souterraine, le 31 mars). Le 16 avril il renouvelle cette confirmation depuis Saint-Émilion, voir Enquête et coutumes Entre-deux-Mers, I-IV ; Gallia christiana, t. II, Inst. col. 291.

${ }^{31}$ Enquête et coutumes Entre-deux-Mers III-16, III-23, V-2, V-20; BRUTAILS Jean-Auguste, éd., Le cartulaire de l'église collégiale de Saint-Seurin, Bordeaux, G. Gounouilhou, 1897, $\mathrm{n}^{\circ}$ 215, ob necessitatem famis propter sterilitatem maximam que anno illo citra solitum modum invaluerat. G. de Nangis : « il y eut en France, et surtout dans l'Aquitaine, une très grande famine, au point que les hommes mangeaient les herbes des champs comme des animaux. Le boisseau de blé valait cent sous dans le Poitou, où un grand nombre de gens périrent de faim ou furent consumés par le feu sacré » (GERAUD Hercule éd., Chronique latine de Guillaume de Nangis de 1113 à 1300 : avec les continuations de 1300 à 1368, Paris, J. Renouard et Cie, Publications pour la société d'histoire de France, 1843, p. 145).

32 Close Rolls 1227-1231, p. 422 De summonicione facienda ad veniendium usque Mirebel. Rex probis hominibus de Sancto Macario salutem. Sciatis quod sumis in expeditione nostra apud Myrabel in obsidione castri de Myrebel. Et ideo vobis mandamus, firmiter injungentes in fide qua nobis tenemini, quatinus sicut nos et honorem nostrum diligitis, visis litteris istis, cuum tota gente vestra illuc a nos ita potenter et honorifice ad servicium nostrum veniatis quod fidelitatem vestram ex eo specialiter debeamus habere commendatam, ipsamque digna remuneratione cum multiplici graciarum actione respicere teneamur. Teste rege apud Mirebel, XXI die julii. Eoddem modo scribitur probis hominibus Regula, Langon', Ryun, Caudroe, Sancte Basilidis, Culture, Vasatensibus, frankesiis de Bezazeis, Bugelun' et Luntrenge, Elye Ridel, Bernardo de Ryons, militibus et aliis probis homiinibus Inter duo Maria.

${ }_{33} \mathrm{RG}, \mathrm{n}^{\circ} 169$ (les arbalétriers sont embarqués sur des galées, RG, $\mathrm{n}^{\circ} 157$ ).

${ }^{34}$ RG, n ${ }^{\circ} 2219$, rex probis hominibus suis de Belin, de Fabba, de Farina, de Marencino et de Marmium, de Gous, de Seignans, de Avena, de Aurea Valle, de Puntuns et de Laluque salutem. Mandamus vobis in fide qua nobis tenemini, firmiter injungentes quod quociens a dilecto serviente nostro Johanne le Parkere fueritis requisiti, prompti sitis et parati ad equitandum et guerreandum una cum eodem Johanne inimicos notros, prout idem Johanne vobis plenius dicet pro parte nostras (...) IX die decembris.
} 
prévôtés de Barsac, de Bazas et de l'Entre-deux-Mers en mars 1274, nul rôle de direction ou de conduite de la troupe n'est attribué aux élites locales. En Entre-deux-Mers, en effet, ce service paraît requis de deux manières. Le cas général, celui de la majorité des paroisses, consiste à répondre à un ordre de mobilisation générale et à limiter, selon les propos des jurés qui déposent en 1237, la participation des paroissiens à un siège, et à condition d'être limité à ce que "les hommes rudes et sans armes peuvent faire »; on pense aux travaux de sape, de terrassement ou à la fabrication de machines de guerre. Mais dans un groupe plus restreint de paroisses et lieux-dits, il s'agit d'envoyer un combattant à l'ost ducal, sans limitation de durée, par une participation financière à ses frais selon le principe des «aidants et des partants » en usage pendant la période carolingienne ${ }^{35}$.

Au total, dans les trois domaines où ils servent de relai aux demandes royales, les prud'hommes sont à un moment ou un autre inévitablement en butte au mécontentement de leurs co-paroissiens, ce qui explique peut-être, pour la levée des questes, le passage d'un système par répartition à un système par quotité, où la quotepart de chacun est définie à l'avance. Ces fonctions leur offrent cependant de quoi conforter des dominations locales, au préalable déjà fondées sur la possession de patrimoines assez importants. Elles les autorisent ainsi à recueillir des informations sur les capacités fiscales de leurs voisins, ce à quoi les aide une connaissance de la valeur des choses et des biens au nom de laquelle leurs expertises sont sollicitées ${ }^{36}$. Ces fonctions leur laissent aussi d'appréciables marges de manœuvre pour établir des compromis, imposer des amendes et prononcer des saisies au détriment de leurs voisins.

Les tensions démographiques, conjoncturelles ou structurelles, n'entrent pas seules en ligne de compte pour expliquer la répétition des démarches collectives de prud'hommes, pour obtenir du roi des confirmations de leurs franchises, pour se plaindre du déficit migratoire ou des exactions qu'ils subissent de la part des baillis. Le resserrement de l'appareil administratif royal, au niveau local avec un plus grand nombre de prévôts, est une tendance lourde depuis les années 1180. Ainsi, alors que les textes ne signalent qu'un prévôt en Bordelais jusqu'à la fin du XII ${ }^{\mathrm{e}}$ siècle, il s'en compte au moins deux de plus vingt ans plus tard : le prévôt d'Entredeux-Mers et celui de Belin ${ }^{37}$. Or, ces nouveaux agents interfèrent doublement sur les prérogatives des prud'hommes. D'abord parce que leurs fonctions militaires et fiscales recouvrent pour partie celles des prud'hommes (ils sont ainsi à plusieurs reprises les destinataires d'ordres de levée des milices villageoises). Ensuite parce que avant les réformes du Prince Édouard, fils d'Henri III, à qui le roi confie la Gascogne après la lieutenance agitée de Simon de Montfort (1248-1253), les prévôts prennent leur charge à ferme du sénéchal qui la cède au plus offrant (non curamus qui ipsi sunt (...) set solum ut carius possit vendere) ( $^{38}$. Les prévôts rentrent ensuite dans leurs frais en multipliant les extorsions sur le dos des populations rurales, prud'hommes compris. C'est aussi pourquoi certains de ces derniers

\footnotetext{
${ }^{35}$ RF, n592, 605, Contamine, Philippe, La Guerre au Moyen Âge, Paris, 1980, p. 101.

${ }^{36}$ Par exemple, $R G, \mathrm{n}^{\circ} 1266$, quod mercandisas contentis in navi predicta (ayant accosté à Bordeaux), que cicius vendi poterunt, per estimacionem proborum et legalium hominum eas vendi faciant; $R G, \mathrm{n}^{\circ} 2644$ quod totam garnesturam vinorum quam rex habet apud Burgum, sine dilatione vendi faciat per visum proborum hominum, et quod de exitibus illius vendicionis pacari faciat municiones castri regis ibidem de quindecim diebus.

${ }^{37}$ En Bordelais, première mention d'un prévôt de l'Entre-deux-Mers, Grand cartulaire de La Sauve-Majeure, éd. Higounet, Ch. et A., Bordeaux, FHSO, 1996, n³7 (1206-1222) ; prévôt de Belin (Patent Rolls 1216-1225, p. 249-252 et 275-276, 1220-1221). Prévôt de Barsac (RG, n 1243).

${ }^{38}$ BEMONT Charles, Simon de Montfort, comte de Leicester, sa vie (120 ?-1265), son rôle politique en France et en Angleterre, Paris, Picard, 1884.
} 
choisissent, plus ou moins volontairement, la protection d'une dépendance renforcée vis-à-vis d'un seigneur, en entrant de la sorte dans la ligesse ou questalité, ces faciès gascons des servitudes que l'on voit être institutionnalisées en de larges parties de l'Occident chrétien de l'époque. Mais d'autres optent pour une autre solution. Les plaintes adressées au roi montrent qu'ils ne restent pas apathiques face à des individus dont les pratiques sapent les fondements de leur autorité traditionnelle. Ils résistent aussi en investissant le terrain des représentations.

\section{Légitimer des dominations contestées}

C'est dans le procès verbal de l'enquête de 1236-1237 que certaines de ces représentations sont formulées le plus clairement sous formes de digressions historiques et informatives sorties de la parole des jurés.

La geste carolingienne est captée : interrogés (requisiti) sur les origines de leurs libertés, les jurés répondent aux deux commissaires royaux que leurs ancêtres, parce qu'ils avaient accompagné Charlemagne dans sa guerre contre les Sarrasins, auraient été gratifiés par la liberté de leurs possessions, en remerciement d'un service gratuit. L'investissement de la geste carolingienne, très populaire en Bordelais, prouve que ces thèmes se diffusent largement au-delà de la société aristocratique ${ }^{39}$. De même, sur le terrain politique, une forme de pactisme transparaît aussi d'après l'affirmation que le pouvoir judiciaire aurait été concédé au duc par les justiciables eux-mêmes, pro pace tuenda. Cette vision d'une autorité publique procédant d'une délégation populaire concerne également les prérogatives du prévôt et celles du sénéchal en temps de guerre. Attestée également plus au sud dans les fors de Béarn, cette vision constitue un marqueur intéressant de la politisation d'une société rurale dont les élites tiennent à souligner leur engagement volontaire dans la sujétion ${ }^{40}$.

Le procès verbal fait aussi fait état de précédents survenus quelques décennies plus tôt pour expliquer l'origine de telle ou telle prestation. Ainsi, sur l'origine de la queste, l'enquête rapporte pas moins de trois traditions expliquant sa mise en place. Elle est signalée parmi les ressources du comte de Poitiers venant en cette terre à l'appel des prélats dépositaires de "l'affaire de paix et de foi », pour lutter contre les bandes de routiers, mais la faiblesse de cet expédient l'aurait amené à demander l'aubergade. On rapporte aussi que la queste aurait été assignée à l'archevêque de Bordeaux par Jean sans Terre pour permettre au prélat de nourrir et de vêtir des pauvres, en compensation d'autres rentes que le roi lui avait assignées. À un autre endroit, il est question d'un précédent remontant à un bailli ducal qui aurait demandé aux hommes de l'Entre-deuxMers la fourniture d'un cheval de quarante livres, une prestation qui aurait constitué un précédent pour les paroisses ultérieurement astreintes au paiement de la queste. Il y a dans ces récits la volonté manifeste d'expliquer les levées qu'ils orchestrent et de parer aux contestations qu'elles occasionnent.

\footnotetext{
${ }^{39}$ Meredith-Jones C. éd., Historia Karoli Magni et Rotholandi ou Chronique du Pseudo Turpin, Paris, 1936 ; MANDACH (de) André, éd., Chronique dite Saintongeaise. Texte franco-occitan inédit "Lee». À la découverte d'une chronique gasconne du XIII siècle et de sa poitevinisation, Niemeyer, Tübingen, 1970 ; CourTEAULT Paul, « Bordeaux et le pays Bordelais dans les chansons de geste », Revue Historique de Bordeaux, mai-juin 1913, p. 161-175.

${ }^{40}$ BARRAQUE Jean-Pierre, « Pactisme et pactismes », in BlAZQUEZ, A. et ChARREYRE Ph., dir., Espaces nationaux et identités régionales. Mélanges en l'honneur du professeur Christian Desplat, Orthez, 2004; $\underline{\mathrm{http}}$ ///halshs.archives-ouvertes.fr/halshs-00290171/en/
} 
Enfin, ces élites se font aussi l'écho d'informations plus contemporaines à caractère politique, circulant au sein de ce milieu social à la manière des rumeurs ${ }^{41}$. Le sénéchal Henri de Trubleville fait ainsi l'objet d'une campagne de dénigrement car on lui prête des propos peu respectueux vis-à-vis du roi. La circulation de ces propos critiques sur le roi au sein des élites rurales est à mettre en relation avec d'autres signes, à la même époque, de l'émergence d'une opinion publique. C'est ainsi que sont vus les pamphlets contre la reine Blanche de Castille, veuve de Louis VIII et mère de Louis IX, accusée de prévarication ${ }^{42}$. En Angleterre, les crises politiques et sociales du règne de Jean sans Terre puis d'Henri III génèrent des prises de position jusqu'au sein de la paysannerie pendant la guerre des barons (1264-1265), si l'on en juge par les participation de groupes de paysans aux combats contre les troupes royales au nom de la «communauté du royaume », un concept politique issu du parti réformateur et manifestement adopté bien au-delà des barons et des clercs ${ }^{43}$.

Tous ces thèmes attestent donc de l'existence auprès des élites rurales d'une forme de mémoire collective mais aussi d'un degré de culture touchant au politique dont les historiens ont jusque là surtout des preuves dans l'aristocratie ou le clergé, mais peu encore auprès des illiterati. S'ils posent des questions sur les modalités de transfert des modèles culturels entre aristocratie, clergé et paysannerie, entre ville et campagnes, s'ils nous interrogent aussi sur le degré de literacy de ces élites paysannes, ces récits et constructions mémorielles ont une fonction évidente. Chacun de ces thèmes permet d'expliquer l'origine de la position sociale des prud'hommes, des prestations dont ils sont les médiateurs ou dont ils collaborent à la collecte, à la manière d'une "dérivation » pour reprendre un concept de Wilfredo Pareto.

\section{Conclusion}

$\mathrm{Au}$ total, ces groupes de notables fonctionnent comme des élites, au sens où l'entend Pierre Bourdieu, puisque leur domination sociale repose sur la possession d'un capital qui n'est pas que matériel. Au sein des ressources immatérielles et symboliques, le capital culturel dont on imagine habituellement les paysans dépourvus est assez important pour être mobilisé afin de défendre ou entretenir une domination locale contestée. Il assoit des logiques de distinction sociale qui conduisent ce milieu à une plus grande proximité avec la petite aristocratie des seigneurs locaux auquel il peut d'ailleurs être relié par des unions matrimoniales. Si l'on peut faire de suggestives comparaisons avec les pratiques des élites bourgeoises qui s'affirment à la même époque, y compris sur le plan culturel, le parallèle s'arrête là, car, derrière leurs enceintes, les bourgeoisies urbaines organisées en communes et municipalités sont en mesure de s'ériger en seigneuries collectives. Dans les campagnes, les groupements de prud'hommes n'en sont pas là. Tout au moins, la contestation de leurs fonctions traditionnelles par une nouvelle génération de relais royaux, accentue-t-elle les logiques de distinction sociale internes aux communautés rurales. L'émergence de la figure du paysan cultivé est donc aussi un effet collatéral de la genèse médiévale de l'état moderne.

\footnotetext{
${ }^{41}$ Boutoulle Frédéric, «"Il est un meilleur roi que le roi d'Angleterre". Note sur la diffusion et la fonction d'une rumeur dans la paysannerie du Bordelais au XIII ${ }^{\mathrm{e}}$ siècle », in BILlORE, M. et SORIA, M., La rumeur au Moyen Âge. Du mépris à la manipulation, $V^{e}$ - $X V^{e}$ siècle, Rennes, PUR, 2011, p. 279-291.

${ }^{42}$ Le GoFF Jacques, Saint Louis, Paris, 1996.

${ }^{43}$ CARPENTER David, «English Peasant in Politics 1258-1267 », in Id., The Reign of Henry III, Londres, 1996, p. 309-348.
} 
